\title{
Aspectos psicossociais do trabalho em Saúde Mental: uma revisão integrativa
}

\author{
Erika Renata Trevisan ${ }^{1}$ \\ Sybelle de Souza Castro
}

Objetivo: analisar as produções científicas nacionais e internacionais que abordem os fatores psicossociais envolvidos na saúde do trabalhador de saúde mental. Método: revisão integrativa nas bases de dados Pubmed, Lilacs e Scielo. A Análise dos dados foi realizada pela codificação e categorização dos conteúdos. Resultados: foram analisados 13 artigos, agrupados e discutidos em três categorias: a satisfação e impacto com o trabalho em saúde mental; qualidade de vida e o sofrimento mental do profissional e estresse em profissionais da saúde mental. Considerações finais: os estudos descreveram, verificaram e caracterizaram aspectos psicossociais envolvidos no trabalho em saúde mental. Espera-se que esse artigo contribua para a valorização da saúde do trabalhador, qualidade de vida, satisfação no trabalho e minimização do impacto do trabalho.

Descritores: Serviços de Saúde Mental; Saúde do Trabalhador; Avaliação de Serviços de Saúde; Qualidade de Vida; Satisfação no Emprego; Impacto Psicossocial.

\footnotetext{
${ }^{1}$ MSc, Professor Assistente, Universidade Federal do Triângulo Mineiro, Uberaba, MG, Brasil.

${ }^{2} \mathrm{PhD}$, Professor, Universidade Federal do Triângulo Mineiro, Uberaba, MG, Brasil.
} 


\title{
Psychosocial aspects of work in mental health: an integrative review
}

\begin{abstract}
Objective: analyze national and international scientific works regarding the psychosocial factors involved in the health of mental health workers. Method: integrative review in the PubMed, LILACS, and SciELO databases. The data were analyzed by coding and categorizing the content. Results: a total of 13 articles were analyzed, grouped, and discussed in three categories: satisfaction with and impact of working with mental health; quality of life and mental suffering of the professional; and stress in mental health professionals. Final remarks: the studies described, verified, and characterized psychosocial aspects involved in working with mental health. This article aims to contribute to the valorization of workers' health, quality of life, and job satisfaction, as well as to the minimization of the impact of the work.
\end{abstract}

Descriptors: Mental Health Services; Occupational Health; Health Services Evaluation; Quality of Life; Job Satisfaction; Psychosocial Impact.

\section{Aspectos psicossociales del trabajo en Salud Mental: una revisión de integración}

Objetivo: Analizar las producciones científicas nacionales e internacionales que aborden los factores psicossociales envueltos en la salud del trabajador de salud mental. Método: Revisión de integración en las bases de dados Pubmed, Lilacs y Scielo. El Análisis de los datos fue realizado por la codificación y categorización de los contenidos. Resultados: Fueron analizados 13 artículos, agrupados y discutidos en tres categorías: la satisfacción e impacto con el trabajo en salud mental; calidad de vida y el sufrimiento mental del profesional y estrés en profesionales de la salud mental. Consideraciones finales: Los estudios describieron, verificaron y caracterizaron aspectos psicossociales envueltos en el trabajo en salud mental. Se espera que ese artículo aporte para la valorización de la salud del trabajador, calidad de vida, satisfacción en el trabajo y minimización del impacto del trabajo.

Descriptores: Servicios de Salud Mental; Salud Laboral; Evaluación de Servicios de Salud; Calidad de Vida; Satisfacción en el Trabajo; Impacto Psicosocial.

\section{Introdução}

A área da atenção em saúde mental passou por diversas e intensas transformações nas últimas três décadas. Aliado à criação do Sistema Único de Saúde (SUS), o movimento de reforma psiquiátrica consolidou mudanças significativas na forma de tratare entender os transtornos mentais. Os desdobramentos dessas transformações podem ser percebidos, principalmente, na criação de serviços substitutivos ao modelo de tratamento hospitalocêntrico, que resultam de um processo histórico de luta pela democratização da saúde no Brasil e busca da superação do modelo de atenção psiquiátrica, pautado na exclusão social, violência e cronificação do sujeito. 
Os pressupostos de Desinstitucionalização e Reabilitação Psicossocial advindos da Psiquiatria Democrática Italiana influenciaram, indiscutivelmente, o movimento social de Luta Antimanicomial no Brasil. Esse movimento exerceu um papel relevante no processo de implementação da Reforma Psiquiátrica, buscando romper com a lógica manicomial da segregação social e da violência, substituindo-a por cooperativas de trabalho e assistência territorial(1).

Dentre as mudanças propostas, destaca-se a substituição progressiva dos leitos psiquiátricos por uma rede de atenção psicossocial que funcione segundo a lógica do território e que garanta, não só o direito à saúde de forma integral, universal e equânime, mas a participação social e a cidadania.

Os Centros de Atenção Psicossocial (CAPS) foram designados como principal referência para o tratamento em saúde mental, definidos por ordem crescente de porte, complexidade, abrangência populacional, horário de funcionamento, número de funcionários e de pessoas atendidas ${ }^{(2)}$. Inicialmente os CAPS apresentavam-se na modalidade CAPS I, CAPS II ou CAPS III, destinados aos adultos com transtornos mentais severos e persistentes, maiores de 18 anos. A partir de 2002, esse modelo foi adotado na estruturação de outros serviços destinados a usuários com necessidades específicas: CAPS i - especializados em crianças e adolescentes com transtornos mentais e CAPS ad - destinado a pessoas que fazem uso abusivo ou são dependentes de álcool e outras drogas.

Sendo assim, foram propostas novas diretrizes políticas para uma organização do trabalho mais horizontalizada, com foco no sujeito e não na doença mental, na busca de possibilidades concretas de exercer a cidadania e estabelecer trocas sociais ${ }^{(3)}$.

Nesse contexto, os CAPS representam a grande aposta da Reforma Psiquiátrica brasileira, no que concerne a atenção integral à saúde mental de crianças, adolescentes, adultos, idosos e devem ser analisadas e compreendidas em sua complexidade.

Nos últimos anos os CAPS cresceram numericamente de forma importante no Brasil, entretanto, poucos estudos consideram questões referentes à relação entre trabalho e saúde nestes serviços ${ }^{(4)}$. Conhecer e entender os fatores psicológicos e sociais do trabalho dos profissionais atuantes na área da saúde mental pode contribuir para novas perspectivas na construção do cuidado no campo psicossocial e na saúde ocupacional destes trabalhadores. Essas questões são relevantes, considerando-se os recursos subjetivos e interpessoais de cada profissional e da equipe multiprofissional fundamentais na produção do cuidado(5).

Devido à sua complexidade, os fatores psicossociais do trabalho, têm sido definidos por vários referenciais teóricos. De acordo com a Organização Internacional do Trabalho $(\mathrm{OIT})^{(6)}$ esses fatores são aqueles que se referem "à interação entre e no meio ambiente de trabalho, conteúdo do trabalho, condições organizacionais e habilidades do trabalhador, necessidades, cultura, causas extratrabalho pessoais e que podem, por meio de percepções e experiência, influenciar a saúde, o desempenho no trabalho e a satisfação no trabalho".

Diante disso, este estudo objetivou analisar as produções científicas de periódicos nacionais e internacionais, publicados nos últimos dez anos, que abordem os fatores psicossociais envolvidos no exercício do trabalho em saúde mental. Esses fatores considerados neste estudo são: satisfação no trabalho, qualidade de vida, impacto do trabalho na vida e na saúde mental do trabalhador.

\section{Método}

Este estudo é uma revisão integrativa definida como uma abordagem metodológica ampla referente às revisões, que permite a inclusão de estudos experimentais e não-experimentais para uma compreensão otimizada sobre o conhecimento atual a respeito da temática analisada. A revisão integrativa identifica, analisa e sintetiza os resultados de estudos independentes sobre o mesmo assunto, contribuindo para o aprofundamento do conhecimento do fenômeno investigado ${ }^{(7)}$.

Para a realização da revisão integrativa, foram executadas as seguintes etapas: elaboração de uma pergunta norteadora; definição dos critérios de inclusão e exclusão dos artigos encontrados; extração dos dados dos artigos selecionados; análise crítica dos estudos e discussão dos resultados, relacionandoos com os referenciais teóricos e apresentação da revisão através da categorização dos dados. Aquestão norteadora foi: Como estão os fatores psicossociais (Satisfação no trabalho, qualidade de vida, impacto do trabalho na vida e na saúde mental) do trabalhador da área de saúde mental que impactam em sua saúde ocupacional?

A coleta de dados foi realizada entre os meses de maio e junho de 2015 e as bases utilizadas foram o Public Medical (PubMed), Literatura Latino-americana em Ciências da Saúde (LILACS) e o portal Scientific Electronic Librery Online (SciELO). Essas bases de dados foram escolhidas pelo fato de serem as 
principais fontes de publicações da área da saúde. Foram utilizados os seguintes descritores controlados pelo DECS e MESH: serviços de saúde mental (SSM); saúde do trabalhador (ST); avaliação de serviços de saúde (ASS); qualidade de vida (QV) satisfação no emprego (SE); impacto psicossocial (IS). Fez-se o cruzamento entre os descritores: SSM/ST/ASS/QV; SSM/ST/ASS/SE; SSM/ST/ASS/IP.

Os critérios de inclusão dos trabalhos encontrados foram: textos em português, inglês e espanhol, disponíveis na íntegra e gratuitamente nas referidas bases de dados; publicados entre janeiro de 2005 e maio de 2015. Foram excluídos desse estudo os artigos em forma de apostilas, cartas e editoriais, pois não contemplam os critérios necessários para pesquisa científica, pois o foco é buscar evidências científicas sobre o tema.

As buscas foram realizadas pelas próprias autoras, utilizou-se um instrumento de extração de dados para revisão integrativa, adaptado por Ursi $(2005)^{(8)}$, o qual contempla as seguintes informações: número do artigo, dados do artigo (Título, ano da publicação, idioma, autores, título do periódico, local do estudo) objetivos, método, resultado, recomendações, identificação de limites e vieses. Os trabalhos selecionados foram identificados, numerados e as informações extraídas a partir do instrumento, foram organizadas em tabelas, com o objetivo de realizar a análise, a partir dos padrões e diferenças identificadas por meio dos processos avaliativos realizados nos estudos.

A análise dos dados obedeceu as seguintes fases: leitura dos resumos de todos os artigos encontrados, de acordo com os critérios de inclusão e exclusão, leitura na íntegra dos artigos da amostra parcial, exploração dos artigos, codificação dos conteúdos emergentes e relevantes, categorização baseada na incidência do conteúdo e nas características dos estudos, conforme proposto por Bardin (2009)(9).

\section{Resultados}

Foram identificados 119 artigos dos quais, após a leitura de títulos e resumos, foram excluídos 103 (87\%), pois não se enquadravam nos critérios de inclusão ou não tinham relação com os profissionais ou com serviços de saúde mental (Tabela 1). Dos $16(13 \%)$ artigos pré-selecionados, após a leitura na íntegra, foram eliminados três $(2,5 \%)$ que não se enquadravam ao tema. Portanto, no total, foram excluídos 106 (89\%) artigos e a amostra final foi composta por 13 (11\%) publicações (Figura 1).

Tabela 1 - Distribuição dos artigos encontrados, excluídos e selecionados nas bases eletrônicas de dados. Período da publicação - janeiro de 2005 a maio de 2015.

\begin{tabular}{lccccc}
\hline $\begin{array}{c}\text { Bases } \\
\text { Bibliográficas }\end{array}$ & $\begin{array}{c}\text { Artigos } \\
\text { Encontrados }\end{array}$ & $\begin{array}{c}\text { Artigos } \\
\text { Excluídos }\end{array}$ & Amostra Parcial & $\begin{array}{c}\text { Excluídos da amostra } \\
\text { Parcial }\end{array}$ & Amostra Final \\
\hline Lilacs & 20 & 17 & 3 & 0 & 3 \\
Pubmed & 87 & 83 & 4 & 0 & 4 \\
Scielo & 12 & 3 & 9 & 3 & 6 \\
Total & 119 & 103 & 16 & 3 & 13 \\
\hline
\end{tabular}

A síntese dos artigos selecionados será apresentada na Figura 1 de acordo com o ano de publicação, título, idioma/país, autores, título do periódico e o tipo de estudo.

\begin{tabular}{|c|c|c|c|c|c|c|}
\hline N & Ano & Título & $\begin{array}{c}\text { Idioma/ } \\
\text { País }\end{array}$ & Autores & Periódico & Tipo de Estudo \\
\hline \multirow{2}{*}{2007} & $\begin{array}{c}\text { Satisfação com o trabalho } \\
\text { e impacto causado nos } \\
\text { profissionais de serviço de saúde } \\
\text { mental. }\end{array}$ & $\begin{array}{c}\text { Português/ } \\
\text { Brasil }\end{array}$ & $\begin{array}{c}\text { Rebouças, D.; } \\
\text { Abelha, L.; } \\
\text { Legay, L. F. }\end{array}$ & $\begin{array}{c}\text { Revista de } \\
\text { Saúde Pública }\end{array}$ & Quantitativo \\
\hline & $\begin{array}{c}\text { Satisfação e sobrecarga de } \\
\text { profissionais de saúde mental: } \\
\text { validade de construto das escalas } \\
\text { SATIS-BR e IMPACTO-BR. }\end{array}$ & $\begin{array}{c}\text { Português/ } \\
\text { Brasil }\end{array}$ & $\begin{array}{c}\text { Bandeira, M. } \\
\text { Ishara, S.A. } \\
\text { Zuardi, W.(10) }\end{array}$ & $\begin{array}{c}\text { Brasileiro de } \\
\text { Psiquiatria }\end{array}$ & Quantitativo \\
\hline
\end{tabular}




\begin{tabular}{|c|c|c|c|c|c|}
\hline 2008 & $\begin{array}{l}\text { O impacto do trabalho em saúde } \\
\text { mental: transtornos psiquiátricos } \\
\text { menores, qualidade de vida e } \\
\text { satisfação profissional. }\end{array}$ & $\begin{array}{l}\text { Português/ } \\
\text { Brasil }\end{array}$ & $\begin{array}{l}\text { De Marco, P. } \\
\text { F.; Cítero, V. A.; } \\
\text { Edilaine Moraes, } \\
\text { E. et al. }{ }^{(11)}\end{array}$ & $\begin{array}{c}\text { Jornal } \\
\text { Brasileiro de } \\
\text { Psiquiatria }\end{array}$ & Quantitativo \\
\hline 2008 & $\begin{array}{l}\text { O trabalho em saúde mental - um } \\
\text { estudo de satisfação e impacto. }\end{array}$ & $\begin{array}{l}\text { Português/ } \\
\text { Brasil }\end{array}$ & $\begin{array}{l}\text { Rebouças, D.; } \\
\text { Abelha, L.; Legay, } \\
\text { L. F. et al..(12) }\end{array}$ & $\begin{array}{l}\text { Caderno de } \\
\text { Saúde Pública }\end{array}$ & Quantitativo \\
\hline 2010 & $\begin{array}{l}\text { O profissional de referência } \\
\text { em Saúde Mental: das } \\
\text { responsabilizações ao sofrimento } \\
\text { psíquico. }\end{array}$ & $\begin{array}{l}\text { Português/ } \\
\text { Brasil }\end{array}$ & $\begin{array}{l}\text { Silva, E.A. } \\
\text { Costa, I.I. }{ }^{(13)}\end{array}$ & $\begin{array}{l}\text { Revista } \\
\text { Latino- } \\
\text { americana de } \\
\text { Psicopatologia } \\
\text { Fundamental }\end{array}$ & Qualitativo \\
\hline 2010 & $\begin{array}{l}\text { Valuación ergonómica de } \\
\text { carga mental y factores de } \\
\text { riesgo psicosocial del trabajo, } \\
\text { en profesionales, técnicos y } \\
\text { administrativos de un centro de } \\
\text { rehabilitación de salud mental } \\
\text { privado. }\end{array}$ & $\begin{array}{l}\text { Espanhol/ } \\
\text { Chile }\end{array}$ & Martínez, R.A.(14) & $\begin{array}{c}\text { Revista } \\
\text { Chilena } \\
\text { de Terapia } \\
\text { Ocupacional }\end{array}$ & Quantitativo \\
\hline 2011 & $\begin{array}{c}\text { BREATHE: a pilot study of } \\
\text { a one-day retreat to reduce } \\
\text { burnout among mental health } \\
\text { professionals. }\end{array}$ & $\begin{array}{l}\text { Inglês/ } \\
\text { Estados } \\
\text { Unidos }\end{array}$ & $\begin{array}{c}\text { Salyers, M.; } \\
\text { Hudson, C.; Gary, } \\
\text { M.; et al. }{ }^{(15)}\end{array}$ & $\begin{array}{l}\text { Psychiatric } \\
\text { Services }\end{array}$ & Quantitativo \\
\hline 2011 & $\begin{array}{l}\text { (In)satisfação com o trabalho em } \\
\text { saúde mental: um estudo em } \\
\text { Centros de Atenção Psicossocial }\end{array}$ & $\begin{array}{l}\text { Português/ } \\
\text { Brasil }\end{array}$ & $\begin{array}{c}\text { Guimarães, } \\
\text { J.M.X. Jorge, M. } \\
\text { S. B.; } \\
\text { Assis, M. M. A. }{ }^{(16)}\end{array}$ & $\begin{array}{l}\text { Ciência } \\
\text { \& Saúde } \\
\text { Coletiva }\end{array}$ & Qualitativo \\
\hline 2011 & $\begin{array}{l}\text { Análise dos níveis de satisfação } \\
\text { de trabalhadores de saúde } \\
\text { mental de um hospital público de } \\
\text { referência psiquiátrica em Belém } \\
\text { (PA). }\end{array}$ & $\begin{array}{l}\text { Português/ } \\
\text { Brasil }\end{array}$ & $\begin{array}{l}\text { Santos, A. M.; } \\
\text { Cardoso, D. A. J.; } \\
\text { Domitila Pereira } \\
\text { Blanco Vieira, D. } \\
\text { P. B. et al.(17) }\end{array}$ & $\begin{array}{c}\text { Revista } \\
\text { Baiana de } \\
\text { Saúde Pública }\end{array}$ & Quantitativo \\
\hline 2011 & $\begin{array}{l}\text { Work as a source of pleasure: } \\
\text { evaluating a Psychosocial Care } \\
\text { Center team. }\end{array}$ & $\begin{array}{l}\text { Inglês/ } \\
\text { Brasil }\end{array}$ & $\begin{array}{l}\text { Glanzner, C. H. } \\
\text { Olschowsky, A. } \\
\text { Kantorski, L.P. }{ }^{(18)}\end{array}$ & $\begin{array}{c}\text { Revista } \\
\text { Escola de } \\
\text { Enfermagem } \\
\text { da USP }\end{array}$ & Quantitativo \\
\hline 2012 & $\begin{array}{c}\text { A saúde mental dos profissionais } \\
\text { de um Centro de Atenção } \\
\text { Psicossocial. }\end{array}$ & $\begin{array}{l}\text { Português/ } \\
\text { Brasil }\end{array}$ & $\begin{array}{l}\text { Athayde, V. } \\
\text { Hennington, E. } \\
\text { A. }{ }^{(19)}\end{array}$ & $\begin{array}{c}\text { Physis Revista } \\
\text { de Saúde } \\
\text { Coletiva }\end{array}$ & Qualitativo \\
\hline 2013 & $\begin{array}{l}\text { Practices in mental health } \\
\text { services: interface with } \\
\text { professionals' satisfaction. }\end{array}$ & $\begin{array}{l}\text { Português/ } \\
\text { Brasil }\end{array}$ & $\begin{array}{l}\text { Macedo, J. Q.; } \\
\text { Lima, H.P.; Alves, } \\
\text { M. D. S. et al. }{ }^{(20)}\end{array}$ & $\begin{array}{l}\text { Text Context } \\
\text { Nursing }\end{array}$ & Qualitativo \\
\hline 2013 & $\begin{array}{l}\text { Recovery-Oriented Services for } \\
\text { Individuals with Mental Illness } \\
\text { and Case Managers' Experience } \\
\text { of Professional Burnout. }\end{array}$ & $\begin{array}{l}\text { Inglês/ } \\
\text { Estados } \\
\text { Unidos }\end{array}$ & $\begin{array}{l}\text { Kraus, S.W. } \\
\text { Stein, C.H. }\end{array}$ & $\begin{array}{l}\text { Community } \\
\text { Mental Health } \\
\text { Journal }\end{array}$ & Quantitativo \\
\hline
\end{tabular}

Figura 1 - Caracterização dos artigos de acordo com o ano de publicação, título, idioma/país, autores, título do periódico e o tipo de estudo. 
Dos 13 artigos selecionados, a maioria foi publicado no ano de 2011 (30,7\%). Prevaleceram os estudos publicados em periódicos nacionais (76,9\%). Quanto à abordagem metodológica dos trabalhos, estes foram classificados como: quantitativos $(n=9$; $69,2 \%$ ) e qualitativos ( $n=4 ; 30,8 \%$ ), prevalecendo o tipo de estudo transversal por meio de aplicação de questionários padronizados, estruturados e validados no país onde o estudo foi realizado.

Os locais onde os estudos foram realizados são muito diversificados, entre os nacionais destacam-se os CAPS, CAPS i, CAPS ad; Programa de Geração de Renda para pessoas com transtornos psiquiátricos; hospital psiquiátrico; hospital geral na unidade psiquiátrica e no departamento de psiquiatria de uma universidade. Quanto às publicações internacionais, os locais são: centro de reabilitação de saúde mental privado e público; instituição para o tratamento da síndrome de Burnout; instituição de saúde mental de longa permanência e serviço de tratamento do uso abusivo de substâncias psicoativas.

A leitura na íntegra dos artigos desta revisão possibilitou o agrupamento dos artigos em três categorias por similaridade de conteúdos que evidenciam os aspectos psicossociais relacionados ao trabalhador da área da saúde mental que influenciam sua saúde ocupacional, São elas: (1) a satisfação e impacto com o trabalho em saúde mental; (2) qualidade de vida e o sofrimento mental do profissional e (3) estresse em profissionais da saúde mental, conforme apresentado na Figura 2.

\begin{tabular}{|c|c|}
\hline Categorias & Título do Artigo \\
\hline $\begin{array}{l}\text { Satisfação e impacto com o } \\
\text { trabalho em saúde mental }\end{array}$ & $\begin{array}{l}\text { 1. Satisfação com o trabalho e impacto causado nos profissionais de serviço de saúde mental. } \\
\text { 2. Satisfação e sobrecarga de profissionais de saúde mental: validade de construto das esca- } \\
\text { las SATIS-BR e IMPACTO-BR. } \\
\text { 4. O trabalho em saúde mental - um estudo de satisfação e impacto. } \\
\text { 9. Análise dos níveis de satisfação de trabalhadores de saúde mental de um hospital público } \\
\text { de referência psiquiátrica em Belém (PA). } \\
\text { 10. Work as a source of pleasure: evaluating a Psychosocial Care Center team. } \\
\text { 12. Practices in mental health services: interface with professionals' satisfaction. }\end{array}$ \\
\hline $\begin{array}{l}\text { Qualidade de vida e sofrimento } \\
\text { mental do profissional }\end{array}$ & $\begin{array}{l}\text { 3. O impacto do trabalho em saúde mental: transtornos psiquiátricos menores, qualidade de } \\
\text { vida e satisfação profissional. } \\
\text { 5. O profissional de referência em Saúde Mental: das responsabilizações ao sofrimento psí- } \\
\text { quico. } \\
\text { 6. Valuación ergonómica de carga mental y factores de riesgo psicosocial del trabajo, en } \\
\text { profesionales, técnicos y administrativos de un centro de rehabilitación de salud mental } \\
\text { privado. } \\
\text { 11. A saúde mental dos profissionais de um Centro de Atenção Psicossocial. }\end{array}$ \\
\hline $\begin{array}{l}\text { Estresse em profissionais da } \\
\text { saúde mental }\end{array}$ & $\begin{array}{l}\text { 7. BREATHE: a pilot study of a one-day retreat to reduce burnout among mental health } \\
\text { professionals. } \\
\text { 13. Recovery-Oriented Services for Individuals with Mental Illness and Case Managers' Ex- } \\
\text { perience of Professional Burnout. }\end{array}$ \\
\hline
\end{tabular}

Figura 2 - Agrupamento dos artigos por similaridade de conteúdo.

\section{Discussão}

\section{Satisfação e impacto com o trabalho em saúde mental}

O trabalho em saúde é um agir tecnológico essencialmente relacional e em ato, onde todo serviço de saúde está vinculado a processos produtivos de atos cuidadores que podem ser "atos cuidadores castradores" ou "atos cuidadores libertadores"(22). Os processos de trabalho em saúde constituemse enquanto lugares de relações, de micropolíticas de encontros e de jogos de poder entre sujeitos e territórios ${ }^{(22)}$. A subjetividade dos trabalhadores 
pode ser tomada como uma das características fundamentais na produção social de saúde mental.

O campo da saúde mental é repleto de especificidades que exigem dos trabalhadores habilidades para lidar com o ser humano e compreendêlo na perspectiva da integralidade do cuidado em saúde. Essa realidade se relaciona diretamente no cotidiano com o sofrimento e a loucura, o que torna o ambiente permeado por intensa produção subjetiva e intersubjetiva. Nesse contexto, os trabalhadores estão expostos a diversas situações, as quais podem ocasionar maior ou menor (in)satisfação(16).

Sendo assim, o trabalhador é o instrumento principal da atenção em saúde mental, que não conta com equipamentos tecnológicos de última geração para auxiliar no cuidado e na atenção integral à saúde dos usuários desses serviços, e frequentemente não dispõe de uma rede assistencial, social e jurídica articulada no sentido da integralidade do cuidado.

Bandeira, et al. (2007) explicita que os profissionais são os agentes que mais sofrem sobrecarga, da mesma maneira que os familiares dos usuários dos serviços de saúde mental. $\mathrm{Na}$ investigação da satisfação e sobrecarga de profissionais de saúde mental, constatou-se que uma avaliação desses trabalhadores se faz necessária, pois ambas as variáveis tem sido consideradas preditivas de qualidade desta desse serviço(10).

Em relação ao impacto do trabalho na saúde dos trabalhadores da equipe de saúde mental, os aspectos que se destacam são: fatores relacionados aos efeitos do trabalho nos aspectos físico e mental, como os problemas físicos descritos pelo trabalhador, consultas ao médico, uso de medicamentos, efeito do trabalho sobre a sua estabilidade emocional e sobre sua necessidade de procurar ajuda profissional; funcionamento da equipe, que podem ser percebidos na frequência com que o profissional pensa em mudar de trabalho, na frequência em que pensa em outro tipo de trabalho que o faria se sentir mais saudável, afastamentos por doença devido ao estresse no trabalho, receio de ser agredido no trabalho, qualidade do sono e saúde em geral; sentimento de estar sobrecarregado, expresso por sentir-se frustrado com os resultados do trabalho, sentir-se sobrecarregado pelo contato constante com os pacientes, sentir-se cansado após o trabalho, sentir-se deprimido por trabalhar com pessoas com distúrbios mentais, sentir-se estressado por trabalhar com doença mental (23)

Os estudos selecionados apontaram que pessoas mais satisfeitas com o trabalho apresentam melhores condições de saúde física e mental e menor ocorrência de doenças, da mesma maneira que maior longevidade. Em contrapartida, os trabalhadores insatisfeitos têm maiores riscos de desenvolver problemas e agravos de saúde. Entretanto, os autores alertam que os resultados devem ser considerados com cuidado, pois pode haver fatores de confusão, que influenciam o nível de satisfação no trabalho e o estado de saúde. Assim, as condições físicas devem ser consideradas covariáveis e não consequências do nível de satisfação no trabalho, visto que, neste momento a associação entre saúde física e satisfação no trabalho é difícil de ser constituída(4).

As relações estabelecidas com os usuários foram relatadas como principal causa de satisfação, enquanto as condições de trabalho e o salário constituem-se nos principais motivos de insatisfação. Além desses aspectos, emergiram consequências na vida dos trabalhadores dos CAPS, particularmente na saúde física e mental ${ }^{(16)}$.

Rebouças, et al. (2008) em seu estudo observou que quanto maior a escolaridade do profissional, menor a satisfação. O impacto do trabalho não está associado com quaisquer das variáveis estudadas por esses autores. Já impacto do trabalho sobre estes profissionais é reduzido e não foram observadas diferenças entre equipes hospitalares e comunitárias ${ }^{(12)}$. A maior parte dos participantes de outro estudo apresentou nível intermediário de satisfação, com a influência da renda mensal pessoal, cargo, turnos de trabalho e impacto do trabalho sobre esses níveis(17).

No processo avaliativo, os trabalhadores demonstraram prazer e realização com seu trabalho narrando sentimentos como orgulho, realização e valorização daquilo que produzem. O prazer pode ser percebido na construção da atenção psicossocial, pois no cotidiano há liberdade para reorganizar o seu trabalho, possibilitando o desenvolvimento de atividades e atitudes capazes de fornecer prazer não profissional de um CAPS ${ }^{(18)}$.

Um dos estudos desta categoria foi qualitativo e teve como objetivo conhecer as percepções e sentimentos dos profissionais sobre a prática em serviços de saúde mental, com foco na satisfação profissional. Os resultados destacaram os impasses vivenciados e os entraves à satisfação profissional, evidenciando o conflito dos trabalhadores frente às exigências do ideário da Reforma Psiquiátrica brasileira, ao qual se sentem aderidos, e a ausência de recursos necessários à sua aplicação, explicitando uma situação conflituosa de lidar com a angústia de não poder fazer seu trabalho, de acordo com o que acreditam que deveria ser feito(20). 
A maioria dos estudos dessa categoria(4) utilizou as escalas de avaliação da Satisfação da Equipe em Serviços de Saúde Mental (SATIS-BR) e escalas de avaliação da sobrecarga do trabalho (IMPACTO-BR) para coleta dos dados. Um dos estudos $^{(10)}$ teve como objetivo avaliar a validade de construto das duas escalas, elaboradas pela Organização Mundial da Saúde (OMS) e adaptadas e validadas para o Brasil por Bandeira et al.. (1999 e 2000)(23). O resultado desta avaliação apontou que as escalas apresentam validade de construto e são adequadas para monitorar a qualidade de serviços de saúde mental. A escala de sobrecarga constituiu um preditor do estresse apresentado pelos trabalhadores em saúde mental.

Qualidade de vida e sofrimento mental do profissional

O trabalho desenvolvido no âmbito da saúde mental, conforme vem acontecendo em vários países do mundo, que propõem mudanças do modelo assistencial, ou seja, a substituição do modelo asilar, pautado na internação manicomial para o modelo psicossocial, baseado no território e na atenção comunitária, exige dos profissionais novas posturas para o ato de cuidar.

Essa transformação da prática do cuidado em saúde mental permite que diferentes concepções e conhecimentos, se constituam na prática interdisciplinar do cotidiano dos serviços de saúde mental apoiada, principalmente, nas experiências e na subjetividade do profissional, através da relação com os usuários.

Sendo assim, o cuidado deixa de ser apenas um procedimento ou uma intervenção e passa a ser uma relação onde a ajuda é no sentido da qualidade do outro de ser ou de vir a ser, respeitando-o, compreendendo-o, tocando-o de forma mais afetiva(24).

Borges, et al. (2002) alertam para os custos emocionais e as necessidades que envolvem a saúde mental dos trabalhadores da área da saúde. Sendo assim, para uma instituição atingir seus objetivos no atendimento e qualidade da atenção prestada, é necessário ter profissionais satisfeitos e que gozem de boa qualidade de vida(25).

Os estudos selecionados e contidos nesta categoria demonstram que os profissionais que atuam na área da saúde mental sentem-se mais satisfeitos em relação à qualidade dos serviços oferecidos e ao trabalho realizado em equipe. O fator que expressa menor satisfação está relacionado as condições de trabalho. De Marco, et al. (2008) observaram em seus estudos escores mais elevados na subescala referente ao impacto emocional, assim como maior presença de transtornos psiquiátricos menores nos profissionais de terapia ocupacional e serviço social, além disso, descrevem que os profissionais que trabalham mais de 20 horas semanais apresentam prejuízos na qualidade de vida(11).

Outro artigo abordou as responsabilizações do profissional de referência em saúde mental, relatadas como geradores de sofrimento psíquico. Entende-se neste caso a referência como um dos dispositivos clínicos do trabalho em saúde mental, que tem como finalidade o estreitamento do vínculo entre os profissionais e usuários, a interdisciplinaridade e a interlocução e articulação com as redes familiares, sociais, culturais, socioeducacionais, entre outros. Os resultados evidenciaram três categorias relacionadas ao sofrimento psíquico: 1) vínculo com usuário e o sofrimento psíquico do referente; 2) organização do trabalho e o papel do referente e 3) suporte institucional e a dimensão clínica no trabalho do profissional de referência(13).

A atuação do profissional de referência em saúde mental exige uma articulação de vários conhecimentos e de diversos campos relacionais, com múltiplas possibilidades de trocas e conflitos ${ }^{(13)}$.

Martínez (2010) realizou um estudo no Chile sobre os fatores psicossociais de risco no trabalho e na qualidade de vida dos trabalhadores de um centro de reabilitação de saúde mental, especificamente os causadores de estresses. Os resultados apontaram que $77,8 \%$ dos participantes da pesquisa percebem seu trabalho com alta carga de trabalho mental. Em relação à avaliação psicossocial, 78,6\% perceberam demandas psicológicas com um elevado nível de risco. Existe uma grande demanda em relação ao trabalhador de saúde que atende pessoas com transtornos psiquiátricos, a forma como o trabalho é sistematizado e a estrutura organizacional são particularmente relevantes, uma vez que a concepção do local de trabalho, a clareza das funções técnicas e profissionais, a pertinência da informação, da equipe, liderança, motivação dos trabalhadores e do ambiente de trabalho influenciam diretamente no impacto do trabalho e na vida dos trabalhadores e da sua saúde, mas também na qualidade da assistência ao paciente ${ }^{(14)}$.

Athayde e Hennington (2012) desenvolveram um estudo com o objetivo de analisar situações concretas de trabalho no CAPS, tendo como foco o processo saúde-doença-trabalho dos profissionais de saúde mental. O método escolhido foi o estudo de caso e as discussões se pautaram nos referenciais teóricos da Ergologia e da Psicodinâmica do 
Trabalho. Os resultados apontaram três categorias analíticas definidas a partir do material produzido por entrevistas: (1) coletivo de trabalho, (2) dinâmica do reconhecimento e (3) sofrimento e defesas. A primeira categoria evidenciou que a atividade cotidiana dos profissionais de saúde mental demanda grande envolvimento subjetivo e revelou-se desgastante, gerando sofrimento e doenças. As condições de trabalho insatisfatórias repercutiram no serviço prestado e na saúde dos trabalhadores. Ainda assim, foi possível identificar potência criativa e aspectos de satisfação pelo trabalho desenvolvido. As principais estratégias utilizadas pelos profissionais para 0 enfrentamento e a superação das adversidades presentes no trabalho foram o apego aos ideais do SUS e da Reforma Psiquiátrica, o suporte da equipe e o reconhecimento do trabalho realizado(19).

\section{Estresse em profissionais da saúde mental}

Os artigos selecionados abordaram também a Síndrome de Burnout em profissionais de saúde mental. Esse conceito se caracteriza como uma síndrome psicológica produzida em resposta aos estressores interpessoais crônicos no trabalho, associado à exaustão de energia decorrente de uma má adaptação a um trabalho estressante, prolongado e com elevada carga tensional, descrevendo uma condição de profissionais cujo trabalho requer elevado grau de contato interpessoal. O Burnout pode manifestar-se através de três aspectos: 1) esgotamento emocional: sentimento de estar sobrecarregado; 2) despersonalização: surgimento de atitude de frieza e distanciamento direcionada aos colegas e ao trabalho; e 3) reduzida realização pessoal: sentimentos de incompetência e falta de produtividade e realização no trabalho(26).

Os dois artigos que abordaram esse tema tiveram estudos quantitativos desenvolvidos nos Estados Unidos. O primeiro estudo teve como objetivo examinar as percepções e a satisfação no trabalho de gerentes de caso de centros comunitários de saúde mental do estado de Ohio. Participaram 114 profissionais e os resultados sugerem que gerentes de casos de saúde mental percebem que quanto mais altos os níveis dos serviços orientados para a recuperação, menores os níveis de despersonalização e desgaste emocional e aumento dos níveis de realização profissional e emprego ${ }^{(21)}$. Embora as variáveis demográficas tenham contribuído para previsão de despersonalização, realização pessoal e os índices de satisfação de trabalho, seus efeitos foram discretos na maioria dos casos. Os resultados sugerem que os profissionais mais velhos estavam mais propensos a relatar maiores sentimentos de orgulho em seu trabalho(21).

O segundo estudo selecionado sobre essa temática teve como objetivo testar uma intervenção para reduzir o Burnout entre profissionais de saúde mental, através de um treinamento para aprender métodos que possam reduzir essa síndrome, em que foram convidados todos os funcionários de um órgão público de tratamento de saúde mental e abuso de substâncias psicoativas ${ }^{(15)}$. $O$ treinamento foi fornecido por meio de oficina de seis horas de duração em um hotel, com 84 participantes composto de uma breve introdução, princípios de prevenção de burnout, exercícios experimentais e habilidade em construção de seis grandes áreas: práticas contemplativas, social, físicas, cognitivo-filosófico, aparência, e outras atividades de autocuidado. Os resultados apontaram queapós seis semanas do treinamento, a equipe relatou diminuições significativas em exaustão emocional e despersonalização e considerável aumento da avaliação positiva dos usuários dos serviços em que trabalham. Não houve mudança relevante em relação à realização pessoal, a satisfação no trabalho ou na intenção de sair dessa posição profissional. Dos profissionais que participaram da intervenção, 91\% declararam que o treinamento foi útil(15).

\section{Considerações Finais}

Nesta investigação foram encontrados estudos que descrevem, verificam e caracterizam os aspectos psicossociais envolvidos no trabalho em saúde mental. Espera-se que os conhecimentos revelados por este estudo contribuam para a criação de espaços para a construção do trabalho que valorize a saúde do trabalhador, a qualidade de vida, a satisfação no trabalho e a minimização do impacto do trabalho na vida desses profissionais. .

Há a necessidade de avançar nos estudos sobre essa temática, considerando-se que desde 2013 não houve mais nenhuma publicação sobre o assunto em periódicos nacionais e internacionais, evidenciando as dificuldades de desenvolvimento de recursos para lidar com as adversidades inerentes ao trabalho em saúde mental.

Estratégias de investigação sistemática em relação à saúde do trabalhador proporcionam reflexões acerca dos processos de trabalho, inclusive possibilitando a elaboração de propostas de intervenção que deem suporte para o profissional de saúde mental, fundamentais para a proteção da saúde desses trabalhadores e, consequentemente, da população atendida. 
Apesar dos critérios usados, essa revisão integrativa da literatura pode apresentar algumas limitações quanto aos descritores utilizados e aos cruzamentos realizados entre eles, podendo ter excluído algum artigo que não estava neste escopo.

\section{Referências}

1. Amarante P. Loucos pela vida: a trajetória da reforma psiquiátrica no Brasil. Rio de Janeiro: SDE/ENSP; 1995.

2. Ministério da Saúde (BR). A política do Ministério da Saúde para a atenção integral a usuários de álcool e outras drogas. [Internet]. Ministério da Saúde; 2003. Disponível em: http://bvsms.saude.gov.br/bvs/ publicacoes/pns_alcool_drogas.pdf

3. Saraceno B. A concepção de reabilitação psicossocial como referencial para as intervenções terapêuticas em saúde mental. Rev Ter Ocup Univ São Paulo. 1998;9(1):26-31.

4. Rebouças D, Legay LF, Abelha L. Satisfação com o trabalho e impacto causado nos profissionais de serviço de saúde mental. Rev Saúde Pública. 2007;41(2):244-50. 5. Porto MF de S, Almeida GES de. Significados e limites das estratégias de integração disciplinar: uma reflexão sobre as contribuições da saúde do trabalhador. Ciênc Amp Saúde Coletiva. 2002;7(2):335-47.

6. Joint ILO WHO Committee on Occupational Health, editor. Psychosocial factors at work: recognition and control. 1. publ. Geneva; 1986. 81 p.

7. Souza MT, Silva MD, Carvalho R. Revisão integrativa: o que é e como fazer. Einstein. 2010;8(1 Pt 1):102-6.

8. Ursi ES. Perioperative prevention of skin injury : an integrative literature review. [Internet]. Universidade de São Paulo; 2005 [cited 2015 May 28]. Available from: http://www.teses.usp.br/ teses/disponiveis/22/22132/tde-18072005-095456/

9. Bardin L. Análise de Conteúdo. Lisboa: Editora 70; 2009. 10. Bandeira M, Ishara S, Zuardi AW. Satisfação e sobrecarga de profissionais de saúde mental: validade de construto das escalas SATIS-BR e IMPACTO-BR. J Bras Psiquiatria. 2007;56(4):280-6.

11. De Marco, Furuta P, Cítero V de A, Moraes E, NogueiraMartins LA. Job impact on mental health workers: minor psychiatric disorders, quality of life and job satisfaction. $J$ Bras Psiquiatria. 2008 Jan;57(3):178-83.

12. Rebouças D, Abelha L, Legay LF, Lovisi GM. Work in mental health: a job satisfaction and work impact study. Cad Saúde Pública. 2008 Mar;24(3):624-32.

13. Silva EA, Costa II da. The reference professional in mental health: from accountabilities to mental suffering. Rev Latinoam Psicopatol Fundam. 2010 Dec;13(4):635-47.

14. Aguirre Martínez R. Evaluación ergonómica de carga mental y factores de riesgo psicosocial del trabajo, en profesionales, técnicos y administrativos de un centro de rehabilitación de salud mental privado. Rev Chil Ter Ocup. 2010;87-99.

15. Salyers MP, Hudson C, Morse G, Rollins AL, Monroe-DeVita M, Wilson C, et al.. BREATHE: A Pilot Study of a One-Day Retreat to Reduce Burnout Among Mental Health Professionals. Psychiatr Serv. 2011 Feb 1;62(2):214-7.

16. Guimarães JMX, Jorge MSB, Assis MMA. (In) satisfação com o trabalho em saúde mental: um estudo em Centros de Atenção Psicossocial. Ciênc Saúde Coletiva. 2011;16(4):2145-54.

17. Santos AM dos, Cardoso DAJ, Pereira Blanco Vieira D, Costa Araújo F, Silva de Farias H, Pelaes da Mota S, et al.. Análise dos níveis de satisfação de trabalhadores de saúde mental de um hospital público de referência psiquiátrica em Belém (PA). Rev Baiana Saúde Pública. 2012;35(4):813.

18. Glanzner $\mathrm{CH}$, Olschowsky A, Kantorski LP. Work as a source of pleasure: evaluating a Psychosocial Care Center team. Rev Esc Enferm USP. 2011 Jun;45(3):71621.

19. Athayde V, Hennington ÉA. The mental health of workers in Psychosocial Care Centers. Physis Rev Saúde Coletiva. 2012 Jan;22(3):983-1001.

20. Macedo JQ de, Lima HP, Alves MDS, Luis MAV, Braga VAB. Practices in mental health services: interface with professionals' satisfaction. Texto Amp Contexto Enferm. 2013 Dec;22(4):999-1006.

21. Kraus SW, Stein $\mathrm{CH}$. Recovery-Oriented Services for Individuals with Mental Illness and Case Managers' Experience of Professional Burnout. Commun Mental Health J. 2012 Mar 22;49(1):7-13.

22. Merhy EE. Cuidado com o cuidado em saúde: saber explorar seus paradoxos para um agir manicomial. In: Merhy EE, Amaral H, editors. A Reforma Psiquiátrica no Cotidiano II. São Paulo: Hucitec; 2007.

23. Bandeira M, Pitta AMF, Mercier C. Escalas brasileira de avaliçäo da satisfaçäo (SATIS-BR) e da sobrecarga (IMPACTO-BR) da equipes técnica em serviços de saúde mental. J Bras Psiquiatria. 2000 Apr;49(4):105-15.

24. Waldow VR. Caring moment: moment of reflection in action. Rev Bras Enferm. 2009;62(1):140-5.

25. Borges LO, Argolo JCT, Pereira A de, Machado EAP, Silva $W$ da. A síndrome de burnout e os valores organizacionais: um estudo comparativo em hospitais universitários. Psicol Reflexão Crítica. 2002;15(1):189-200. 26. Maslach C, Schaufeli WB, Leiter MP. Job Burnout. Annu Rev Psychol. 2001;52(1):397-422. 